Journal of Food and Nutritional Sciences Research (JFNSR) 2021

Volume 3, Issue 1, Pages 169-205

https://doi.org/10.37512/1400

Open Access - Published by the Kenya Institute of Food Science and Technology

Original Review Article

\title{
Roles of Lipids in Health and Disease.
}

Corresponding author: Michael N.I. Lokuruka

Department of Food Science and Nutrition, Karatina University, P.O Box, 1957-10101,

Karatina, Kenya. e-mail: mlokuruka@karu.ac.ke and mlokuruka@gmail.com

Received 7.7.2021, Reviewed 15.12.2021, Accepted 23.12.2021

\begin{abstract}
This literature review is an update of the functions of lipids, with emphasis on their roles in health and disease conditions. Fats transport micronutrients and phytochemicals, thus raising their bioavailability. Fatty acids are needed to maintain brain integrity and function with omega-3 fatty acids being necessary for brain development during fetal and postnatal period upto about 2 years of age. Decosahexaenoic acid is required for optimal maturation of the retina and visual cortex, with visual acuity and mental development seemingly improved by extra DHA. Lipids regulate cell signalling, gene transcription and expression and govern all immune processes. Bioactive lipid mediators are generated from PUFAs, especially EPA, DHA and DPA. Eicosapentaenoic acid promotes antiplatelet aggregation and vasodilation, is anti-inflammatory and maintains plasma triglyceride levels low. Decosapentaenoic acid enables alterations in membrane structure and function, influences membrane protein function, cellular signalling and lipid mediator production. Low DHA levels have been linked to poor cognitive development and visual function, while DPA reduces platelet aggregation, improves lipid metabolism, endothelial cell migration, and resolution of chronic inflammation. Omega-3 PUFAs minimize bone mineral loss in old age and may prevent age-related macular degeneration and dry eye disease. However, the association of bone mineral density with n-3 fatty acid intake requires further research. Although immunomodulation through the essential fatty acids has shown promising results in the potential for lipids as therapeutic molecules, the mechanisms of action require further investigation. Lipid ratios are proving to be useful biomarkers for CVD, Alzheimer's disease and diabetes.
\end{abstract}

KEYWORDS: Lipids, eye, bone, brain, health, disease. 


\section{INTRODUCTION}

Lipids are organic, nonpolar, heterogeneous, oily/greasy compounds that are soluble in nonpolar solvents and insoluble in water, because water is a polar molecule.

Among the different classes of lipids are fatty acids, triglycerides (TG), glycerophospholipids, sphingolipids, isoprenoids and steroids. These components are also broadly classified as either simple or complex lipids. Simple lipids include fatty acids, fats and oils, sterols and waxes, while complex lipids include glycolipids, phospholipids, lipoproteins, sulfolipids and aminolipids. While saturated fatty acids (SAFAs) contain no double bonds, polyunsaturated fatty acids (PUFAs) and monounsaturated fatty acids (MUFAs) contain at least two double bonds and one double bond, respectively.

\section{LIPID METABOLISM}

Most food lipids are taken into the human body in the form of TGs and cholesterol. Their metabolic bioconversion begins in the intestine, where ingested TGs are broken down to fatty acids (FAs) and monoglyceride molecules by pancreatic lipases (lipolysis), enzymes that break down fats after emulsification by bile salts. When food reaches the small intestine in the form of chyme, the hormone, cholecystokinin is released by intestinal endocrine I-cells in the intestinal mucosa and in cerebral neurons (Rehfeld, 2017). Cholecystokinin stimulates the release of pancreatic lipase from the pancreas and stimulates the contraction of the gall bladder to release stored bile salts into the intestine. It also travels to the brain, where it can act as a hunger suppressant (Rehfeld, 2004). The free fatty acids and monoglycerides are transported into the enterocytes (the major intestinal epithelial cells),-which are packaged along with cholesterol molecules in lipoprotein vesicles referred to as chylomicrons. The chylomicrons enable fats and cholesterol to move within the aqueous environment of the human lymphatic and circulatory systems, from where most of the triglyecerides are taken up by adipose tissue and skeletal muscle, while the cholesterol-rich chylomicron remnants are taken up by the liver. Despite the exogenous source of FAs as explained above, de novo synthesis also occurs in the liver and adipose tissue (de Carvalho and Caramujo, 2018). Further, lipid metabolism involves the synthesis of the structural and functional lipids (such 
as phospholipids, glycolipids, sphingolipids, cholesterol, prostaglandins, etc.) that are characteristic of individual tissues and the degradation of lipids to satisfy the metabolic needs of the body. Lipid metabolism is in a constant state of dynamic equilibrium. It is a balance between lipid synthesis (lipogenesis) and degradation (lipolysis and oxidation), which determines fat mass. Over $90 \%$ of total energy reserves are stored in adipocytes, mainly as TG that can be hydrolyzed (lipolysis) following hormonal stimulation to release FAs. Fatty acids have two possible fates: $\beta$-oxidation to produce ATP, or re-esterification back into TG. Studies have shown that the hormone leptin has a direct autocrine or paracrine mode of action on lipid metabolism (Vairappan, 2016; Stern et al., 2016). It inhibits the expression of Acetyl-CoA carboxylase (ACC) (Oh et al., 2005), a rate-limiting enzyme for long chain FA synthesis, and is essential for the conversion of carbohydrates to FA, and caloric storage as TG. This stimulation of FA oxidation is probably the key event for the tissue lipid lowering and insulin-sensitizing effects of leptin. This at least partly stimulates adenosine monophosphate and this inactivates ACC, and decreases MalonylCoA concentration, thus stimulating Carnitine palmitoyltransferase 1 (CPT 1) mediated FA oxidation in the mitochondria (Scaglia et al., 2009).

Lipid metabolism and the-accumulation of oxidized of low-density lipoproteins (LDL) are the basis for the development of atherosclerosis. Macrophages internalize excessive amounts of oxidized LDL, become enlarged and full of lipids, and then transform into foam cells. Parts of the foam cell and dying macrophages accumulate in vessel walls, thereby participating in atherosclerotic plaque formation. Reduced levels of high-density lipoprotein (HDL) cholesterol together with high levels of LDL are known risk factors for the different maladies that involve lipids. High-density lipoprotein is involved with the transport of excess cholesterol from macrophages and other cells to the liver, so it is vital for diminishing the accumulation of foam cells in atherosclerosis.

Other functions of lipids are discussed below.

\section{Lipids in Insulation and Organ Protection}

Up to $30 \%$ of a person's body weight is comprised of fat tissue (Lorenzo et al., 2016), with some of it being made up subcutaneous and visceral fat or adipose tissue surrounding and 
protecting delicate internal organs, such as the heart, kidneys and liver. Subcutaneous fat insulates the body from extreme temperatures and helps keep the internal climate under homeostatic control. It pads hands and buttocks and prevents friction, as these areas frequently come in contact with hard surfaces. It therefore gives the body the extra padding required when engaging in physically-demanding activities such as soccer, horse riding and rugby playing where athletes experience frequent knocks and shocks.

\section{Lipids for brain function and integrity}

The composition of the brain is outstandingly $60 \%$ fat, demonstrating the major structural role that fat serves within the brain (Chang et al., 2009). The brain is a key organ for the regulation of energy homeostasis (Shin et al., 2009). Fatty acids are among the most crucial molecules that determine the brain's integrity and ability to perform its functions. Essential fatty acids (EFAs) are required for the maintenance of optimal health, but they cannot be synthesized by the body and must be obtained from dietary sources. Clinical observational studies have related imbalanced dietary intake of FAs to impaired brain performance and diseases. The EFAs, particularly the omega-3 FAs, are important for brain development during both the fetal and postnatal period up to about 2 years of age (Bourre et al., 2004; Leyrolle et al., 2021). Decosahexaenoic acid (DHA) is needed for the optimum functional maturation of the retina and visual cortex, with visual acuity and mental development seemingly improved by extra DHA (Walchuk and Suh, 2020). Beyond their important role in building the brain structure, EFAs, as messengers, are involved in the synthesis and functions of brain neurotransmitters (Yehuda et al., 2005), and in the molecules of the immune system (Guiterrez et al., 2019). Neuronal membranes contain phospholipid pools that are the reservours for the synthesis of specific lipid messengers on neuronal stimulation or injury. These messengers in turn participate in signaling cascades that can either promote neuronal injury or neuroprotection (Sambra et al., 2021). Homeostasis ultimately ensures a remarkably stable body weight throughout the adult lifespan. 


\section{Lipids in Vitamin Absorption and Nutrient Bioavailability}

The dietary fats in foods transport micronutrients, thus raising their bioavailability through improved intestinal absorption. Fat-soluble nutrients are especially important for good health and exhibit a variety of functions. Vitamins A, D, E, and K-the fat-soluble vitamins, are mainly found in foods containing fat. However, some fat-soluble vitamins, such as vitamin A are also found in naturally fat-free foods such as green leafy vegetables, carrots, and broccoli. These vitamins are best absorbed when combined with foods containing fat. Fats also increase the bioavailability of phytochemicals (Sophie et al., 2017), which are plant constituents that include lycopene (found in tomatoes) and betacarotene (found in carrots and yellow and green vegetables). Beta-carotene is the precursor of Vitamin A. Phytochemicals promote health and well-being (Yoo et al., 2018). As a result, eating tomatoes or green leafy vegetables with olive oil or salad dressing should facilitate better lycopene and beta-carotene absorption. Other essential nutrients, such as the EFAs, are constituents of the fats themselves and serve as building blocks of body cells. Of particular importance in food lipids are the omega- 3 and omega- 6 fatty acids, with the former predominating in seafood and particularly as components of fish oils. They are also found in plant seeds that are major sources of edible oils for human nutrition. Excellent food sources of omega-3 FAs include: salmon, flaxseed and walnuts, while good sources include: scallops, cauliflower, cabbage, cloves and mustard seed (Prakash, 2020). Any food processing procedure that removes the lipid elements from food, also invariably takes away the food's fat-soluble vitamins. When products such as grain and milk are processed, either by way of physical removal of fat or the fat-containing components, these essential nutrients are lost. Manufacturers have therefore to replace these nutrients through enrichment of the processed food product.

\section{Lipids as Disease Biomarkers}

Quantitation of changes in the lipidome of a human cell, tissue, organ or biofluid in health and disease, has been identified recently using high resolution mass spectrometry, to change with the health and disease condition of a patient (Ghosh and Nishtala, 2017). It therefore appears that the lipidome of a particular organism has relevance to the disease 
manifestation as it reflects the metabolic changes which can be a consequence of the disease. Hence these changes in the molecules can be considered as potential markers for screening and early detection of the disease (Ghosh and Nishtal, 2017). Lipid ratios have been proven to be a useful biomarker for several diseases, including cardiovascular disease, Alzheimer's disease, and diabetes (Dhama et al., 2019). In addition, using lipid ratios as a disease indicator would carry more information and be more biologically meaningful. The results of several studies showed that the ratio of glycerophospholipids/sterol lipids were lower in congestive pulmonary disease patients than in healthy controls (Liu et al., 2020), as well as in brain disorders (Hussain et al., 2019), cancer and bacterial infections (Larrouy-Maumus, 2019) and Alzheimer's disease (Zarrouk et al., 2018). In a meta-analysis using data from 6 countries, available studies of free-living populations globally, biomarker concentrations of seafood and plant-derived $\omega$ 3 fatty acids were shown to be associated with a lower incidence of fatal coronary heart disease (CHD) (Del Gobbo et al., 2016).

\section{Role of Lipids in Regulation and Impulse Signalling}

Fats play important functional roles in sustaining nerve impulse transmission (Panov et al., 2014), memory storage and tissue structure (de Carvalho and Caramujo, 2018). More specifically in the brain, lipids are critical to brain activity in structure and in function. They help form nerve cell membranes, insulate neurons and facilitate the signaling of electrical impulses throughout the brain (Bruce et al., 2017). Cellular membranes are assembled by amphipathic lipids and proteins. Because these associations are affinity-based, membranes are considered fluid, allowing rotational, translational and transbilayer lipid movement (Goni, 2014).

Lipids found in the brain are grouped as sphingolipids, glycerophospholipids, and cholesterol, and are considered to be present in almost equal ratios (Zhang and Liu, 2015). These lipids are involved in developmental, maintenance and many other cellular processes of the brain. The lipids act as signaling molecules, sources of energy, contributing to synaptogenesis, neurogenesis, and impulse conduction (Hussain et al., 2015; Cermanati et al., 2015). Furthermore, lysophospholipids, endocannabinoids, and sphingolipids have also been reported to be involved in cellular signaling, including the regulation of 
numerous ion pumps, channels, and transporters (Hardie and Muallem, 2009; Ridone et al., 2015). All vital events responsible for the development and maintenance of functional activities of the nervous system, depend on the unique lipid contents found in the different membrane regions (lipid rafts) of neuronal cells.

Lipid composition confers important properties on the plasma membrane (PM), with asymmetry, due largely to enriched varieties of lipids within distinct membrane leaflets. In mammalian cells, the PM cytoplasmic leaflet usually contains more phosphatidylethanolamine and phosphatidylserine than the outer leaflet which is rich in sphingolipids (de Kroon et al., 2013). Similar to the above other phospholipids, phosphatidylcholine can transduce cell signal responses not only by acting as membrane scaffold proteins, but also by modulating the subcellular distribution of key signaling regulators (van der veen et al., 2019). In addition, lipids can exhibit different overall geometric shapes based on inherent properties, such as head group size and the length and/or saturation of their acyl chain tails (Rinaldin et al., 2020). Maintenance of membrane fluidity, despite the presence of saturated lipids, is sustained by integrated sterols that interfere with acyl chain packing (Hussain et al., 2015; Cermanati et al., 2015). Conversely, because sterols themselves are inflexible, they can increase membrane rigidity if associated with flexible unsaturated lipid bilayer (Sunshine \& Iruela-Arispe, 2017). Cholesterol deposition can similarly be asymmetrical and differs greatly among organelles (Sezgin et al., 2017). Furthermore, both saturation states and the presence of cholesterol are sufficient to prevent lipid miscibility and lead to specific lipid domains formed by associations of liquid ordered and disordered phases (Goni et al., 2014). These physical properties can act as the template for membrane curvature, thickness, and tension, which play important roles in cell signaling. Furthermore, membrane lipids are known to act as secondary messengers during cell signaling. Many G-protein coupled receptors activate the phosphatidylinositol signaling pathway, whereby phospholipase C hydrolyzes phosphatidylinositol 4,5-biphosphate (PIP2) into two secondary messengers: inositol 1,4,5-triphosphate (IP3) and diacylglycerol (DAG) (Bae et al., 1998). In turn, DAG activates protein kinase $\mathrm{C}$ and IP3 increases intracellular calcium levels, thus promoting many cellular responses, including transcription, cell growth and immune responses. Moreover, 
the lipid sphingosine has been shown to modulate protein kinase C (Hannun et al., 1989). Therefore, membrane lipids alter integral membrane receptor signaling either through direct or indirect stoichiometric interactions. Investigations have identified important roles of lipids in the regulation of membrane protein receptors during cell signaling (Sunshine and Iruela-Arispe, 2017). It is well accepted that the composition of distinct phospholipids in eukaryotic membranes is essential for maintaining cellular homeostasis (Bohdanowicz and Grinstein, 2013). Furthermore, the macrodomain heterogeneity caused by lipid phases can promote segregation and crowding, that, in turn, limits lateral diffusion in a manner that affects cell signaling by either facilitating or hindering protein-protein interactions (Goni et al., 2014). In addition, the local constituency of phospholipids has recently been shown to impact signaling by regulating the exposure of specific protein domains to the cytosol (Li and Buck, 2017).

Lipid composition affects how signaling proteins associate with the PM. A recent study has uncovered that the function of K-Ras can be greatly influenced by the composition of phospholipids in the PM as they offer distinct affinity to the protein (Li and Buck, 2017). Ras, a small GTPase that regulates multiple signaling pathways and is known to be linked to several cancers, is only fully functional once bound to the PM. Other signaling pathways including phosphoinositide 3-kinase/mammalian/mechanistic target of rapamycin (PI3K/mTOR) and the mitogen-activated protein kinase (MAPK) play significant roles in regulation and signaling (Roux and Topisirovic, 2012, 2018)

In addition to their multiple roles in providing unique and critical physical and chemical properties to plasma membranes, lipids have now emerged as important regulators of cell signaling. Their new roles are mediated either by their association with receptors and secondary messengers, as direct ligands to receptors, or by regulating the assembly of complex signaling networks.

\section{Lipids and Gene Expression}

Gene expression is the synthesis of a functional gene product using the information provided by deoxyribonucleic acid-DNA (Perdew et al., 2006). Ribonucleic acid (RNA) is synthesized from DNA through the process of transcription, which is part of the process of gene expression (Singh et al., 2018). As an organism develops or responds to changes in its 
environment, cells can adjust the type and amount of gene expression (Singh et al., 2018). However, the role and nature of the lipids that take part in gene expression and the mechanisms of action is less understood. But it is likely that the role in gene expression is related to the plasma levels of n- 6 and n-3 FAs and the SAFA to PUFA ratio (Ebrahimi et al., 2019). In humans, linoleic acid (LA; 18:2n-6) and alpha-linolenic acid (ALA; 18:3n-3) are not biosynthesized de novo (Burdge and Calder, 2005). Since the conversion of these FAs into long-chain PUFAs is limited, plasma PUFA levels have been shown to be objective biomarkers of dietary intake (Astorg et al., 2008). Hence, using plasma FAs to examine the association between dietary fat quality and gene expression has lately been undertaken to try to understand the role of FAs in gene expression. The ability of FAs to regulate gene transcription may account for their effects on lipid metabolism. Also, it is likely that PUFAs affect gene expression through various mechanisms including, but not limited to changes in membrane composition, intracellular calcium levels and eicosanoid production (Sampath and Ntambi, 2005). Furthermore, PUFAs and their various metabolites can also act at the level of the nucleus, in conjunction with nuclear receptors and transcription factors, to affect the transcription of a variety of genes (Sampath and Ntambi, 2005). Afman and Muller (2012) and Desvergne et al. (2006) suggest that FAs regulate gene transcription directly by binding as ligands to specific transcription factors, thereby controlling the activity of the transcription factor, or indirectly by regulating different signaling pathways controlling the nuclear abundance of transcription factors. Also, there is emerging evidence that PUFAs modulate the transcription of genes involved in lipid metabolism by regulating the activity of the nuclear receptors peroxisome proliferator activated receptors (PPAR) and Liver X Receptor (LXR) or by suppressing the nuclear abundance of the sterol regulatory element binding protein (SREBP) (Afman and Muller, 2012; Giorgidi and Kersten, 2012). However, how SAFAs modulate the expression of genes encoding proteins related to lipid metabolism is not yet clear. But as changes in gene expression occur prior to changes in protein levels, gene expression analysis may therefore be a valuable tool to measure early changes related to diet (de Mello et al., 2012; Afman et al., 2014). Peripheral blood mononuclear cells (PBMCs) include lymphocytes and monocytes which circulate around in the body and are exposed to both environmental factors and metabolic tissues. 
They have therefore been used recently as a surrogate model for liver metabolism as these cells reflect hepatic regulation of cholesterol metabolism, and the extent of metabolic and immune responses (Bouwens et al., 2007; Caimari et al., 2010; Myrhstad et al., 2011). In a study, Liu et al. (2014) investigated the molecular mechanisms of butyrate (as butyrate glycerides, BG)-induced lipid metabolism at the level of gene expression in the jejunum and liver of broilers. The results demonstrated that BG reduces body fat deposition via regulation of gene expression, which is involved in the biological events relating to the reduction of synthesis, storage, transportation and secretion, and improvement of oxidation of lipids and FAs. So butyric acid, a small molecule, also takes part in gene expression as a saturated fatty acid, thus demonstrating the general nature of gene expression by FAs, irrespective of molecular weight and nature. Also, Bouwens et al. (2009) examined the effects of supplementation with the PUFAs, eicosapentaenoic acid (EPA) and DHA on whole-genome PBMC gene expression profiles, in healthy subjects, in a doubleblind trial by using whole-genome transcriptomics analysis. The results showed that fish oil supplementation alters lipid metabolism and increases the proportion of phospholipids and TGs containing long-chain PUFAs. Larsen et al. (2018) established that PBMCs express genes involved in hepatic lipid metabolism and that the expression of several of the genes was influenced by plasma FA levels. The study therefore seems to support the use of PBMCs as a model system for investigating the role of dietary n-3 and n-6 FAs on gene expression related to lipid metabolism. The plasma SAFA to PUFA ratio seems to be more important than the plasma n-6 and n-3 FA level alone with regard to influencing mRNA levels (Larsen et al., 2018).

\section{Transcription Factor Activity}

A considerable number of enzymes and transcriptional factors play key roles in lipogenesis and lipolysis including peroxisome proliferator-activated receptor $\beta$ ( $\beta$ PPAR), peroxisome proliferator-activated- $\alpha$ (PPA- $\alpha$ ), hormone sensitive lipase (HSL), lipoprotein lipase, fatty acid binding protein 3 (fabp3), and malic enzyme (Kiens et al., 1985; Jenkins-Kruchten et al., 2003; van Raalte et al., 2004; Poulsen et al., 2012 ). Others include: liver x receptor (LXR), hepatocyte nuclear factor (HNF)-4alpha (HNF-4) $\alpha$, nuclear factor-kappaB 
(NFkappaB or NFkB), and sterol regulatory element binding protein (SREBP) (OlivaresRubio and Vega-Lopez, 2016; Ayisi et al., 2017). SREBP helps maintain proper lipid levels in the cell, while LxR and NFKB regulate cholesterol and lipid metabolism in mammals and innate immunity, respectively. Although a detailed description of the roles and mechanism of action these factors is discussed in several other sources (Zheng et al., 2013; OlivaresRubio and Vega-Lopez, 2016; Ayisi et al., 2017), they all play key and complementary roles in lipid homeostasis; however, a key factor in the homeostatic control is HSL, which promotes lipolysis in adipocytes and lipid droplets and so modulates the rate of lipid mobilization (Kiens et al., 1985). The liver is the major organ for both the lipolysis and lipogenesis of lipids in human metabolism. The liver utilizes a set of hepatic transcription factors to regulate the expression of genes implicated in all aspects of lipid metabolism including catabolism, transport, and synthesis. The principal regulatory pathways are composed of simple modules of transcription factor crosstalks, which correspond to building blocks of more complex regulatory networks. These transcriptional networks contribute to the regulation of proper lipid homeostasis in parallel to posttranslational mechanisms and end product-mediated modulation of lipid metabolizing enzymes (Karagianni and Talianidis, 2015). It is generally acknowledged that fabp3 plays an intermediate role in orchestrating the gene transcription process involved in lipid homeostasis (Lu et al., 2014), as it is responsible for the intracellular transport of FAs, and together with LA, cooperatively modulate the growth of hepatocytes. Fatty acid effects on gene expression seem to be cell-specific and are influenced by fatty acid structure and metabolism. Fatty acids interact with the genome through several mechanisms. They regulate the activity or nuclear abundance of several transcription factors, including peroxisome proliferator activated receptor alpha (PPAR $\alpha$ ), liver $x$ receptors (LXR), hepatocyte nuclear factor (HNF)-4alpha (HNF-4) $\alpha$, nuclear factor-kappa nuclear factorkappaB (NFkappaB) or NFkB), and sterol regulatory element binding protein (SREBP) (Brown and Goldstein, 1997; Karpen, 2002; Sampath and Ntambi, 2005; Bougarne et al., 2018; Jump, 2018). Fatty acids or their metabolites bind directly to specific transcription factors to regulate gene transcription. Alternatively, FAs indirectly act on gene expression through their effects on, a) specific enzyme-mediated pathways, or b) pathways that 
involve changes in membrane lipid/lipid raft composition that affect G-protein receptor or tyrosine kinase-linked receptor signaling (Jump, 2008). The cell-specific context and the particular gene targeted determine the mechanism of control (Jump, 2008). However, there is some controversy about the involvement of some nuclear receptors, despite PPARs being the preferred receptors for fatty acids, especially PUFAs. The PPARs carry out complementary and sometimes overlapping functions in the control of glucose and lipid metabolism in many tissues (Bravo-Ruiz et al., 2021). This could be one of the reasons why drugs targeting these factors have adverse effects in some cases and undetermined in others and still do not represent the coveted magic bullet against obesity and type 2 diabetes mellitus. Perhaps combinatorial strategies or supplementation with unsaturated fatty acids, which are natural ligands of the PPARs, could be safer and more effective drugs or nutraceuticals (Bravo-Ruiz et al., 2021).

\section{Lipids in Regulation of Production of Bioactive Mediators}

Bioactive lipid products of lipid metabolism play active roles in regulating cellular functions. These bioactive lipid mediators include FAs and their metabolic products, acylglycerol derivatives, endocannabinoids, lysophospholipids, sphingolipids, and cholesterol metabolites. A considerable number of lipid mediators are produced in obese adipose tissue (Cläria et al., 2013). Bioactive lipid mediators are generated from PUFAs, especially those that are abundant in fish lipids-EPA), DHA and decosapentaenoic acid (DPA) (Vacharajani et al., 2009). These molecules also play important roles in adipose tissue functioning (Vacharajani et al., 2009), with the omega-3 mediators promoting the resolution of adipose tissue inflammation (Lopategi et al., 2016), and probably preventing comorbidities related to obesity (Liu et al., 2013). A wide network of soluble mediators, among which endogenous bioactive lipids, governs all immune processes. They are secreted by basically all cells involved in inflammatory processes and constitute the crucial infrastructure that triggers, coordinates and confines inflammatory mechanisms (Kumar et al., 2014). However, mediators formed from omega- 6 FAs are involved in the detrimental transition from acute to chronic inflammation, be it for persistent or excessive action of pro-inflammatory lipids or for the impairment of the functions carried out by resolving ones (Serhan, 2014). Bioactive lipids have been linked to several chronic diseases, 
including rheumatoid arthritis (Valner et al., 2021), atherosclerosis (Linton et al., 2019), diabetes (Natarajan and Nadler, 2004), cancer (Cruz et al., 2020), inflammatory bowel disease (Alhouayek et al., 2021), systemic lupus erythematosus (Navarini et al., 2018), and multiple sclerosis (Hayes and Ntambi, 2020; Ruiz et al., 2019). It has been shown that distinct anionic lipids modulate binding and cytosolic orientation of K-Ras4A, such that the availability of its catalytic domain and ability to interact with effector molecules relies on its interaction with membrane lipids (Sunshine and Inuela-Arispe, 2017). In the inflammatory process, eicosanoids are the fire-starters of the inflammatory processes and engage the mainly cells of the innate arm of immunity that execute all possible strategies to quickly eradicate the injury (Shimizu, 2009; Serhan, 2014). If the danger ceases or is successfully terminated, the fire of inflammation is elegantly extinguished by SPMs that recruit non-phlogistic innate immune cells and activate resolution pathways, aimed at healing the damaged tissue (Basil and Levy, 2016).

At the peak of acute inflammation the same cells producing pro-inflammatory lipid mediators switch function and start producing specialized proresolving lipid mediators (SPMs) from $\omega$-6 FAs and even more from $\omega$-3 PUFAs, EPA, DHA, and DPA, through the stero-selective and concerted action of the same enzymes engaged in classical eicosanoids production: cyclooxygenase, lipoxygenase, and P450 (Chiurchiu et al., 2018).

But if the injurious stimulus is either persistent or not eliminated, perhaps by failure of resolving inflammation, a wildfire of long-lasting inflammatory processes occurs and the flame of inflammation is kept alive mainly by cells of the adaptive arm of immunity, thus leading to many chronic inflammatory diseases. Under the circumstances, tissues activate several adaptation mechanisms that allow cells to cope with the changes induced by the damage, and also due to other families of bioactive lipids like lysophospholipids, sphingolipids, and endocannabinoids, that ultimately regulate cell growth, differentiation, and destiny with the goal of helping the body to restore homeostatic balance (Chiurchiu et al., 2016). Most of these bioactive lipids and several elements of their complex metabolism and signaling (i.e., enzymes and receptors) are differentially dysregulated in many chronic inflammatory diseases (Chiurchiu et al., 2018). 


\section{Omega-3 Lipids in Bone Health}

As dietary lipids may be important, especially for bone integrity in old age, studies of elderly people are warranted (Corwin, 2003). Recent investigations indicate that the type and amount of PUFAs influence bone formation in animal models and osteoblastic cell functions in culture. In growing rats, supplementing the diet with omega-3 PUFA resulted in greater bone formation rates and moderates ex-vivo prostaglandin E(2) production in bone organ cultures (Watkins et al., 2003). A protective effect of omega-3 PUFA on minimizing bone mineral loss in ovariectomized rats was also been reported. The actions of omega-3 fatty acids on bone formation appeared to be linked to altering osteoblast functions (Watkins et al., 2003). A study by Jarvinen et al. (2012) suggests a positive relationship between the dietary PUFAs and bone mineral density (BMD) at lumbar spine and in total body, but not at femoral neck. Further analyses revealed that these results were due to associations among the women without hormone therapy (HT) at baseline. Among them, the intake of total PUFAs as well the intakes of linoleic and linolenic acids and total n-3 and n-6 fatty acids were significantly associated with BMD at lumbar spine; $P$ for trend over the quartiles ranged between 0.013 and 0.001. Similarly, significant associations were demonstrated for total body BMD and fatty acids with an exception of total PUFA. No significant associations were found among women with HT at baseline (Jarvinen et al., 2012). Because of differences in the quantity and quality in dietary PUFA among different populations this relationship is not unequivocal and further studies in populations with different dietary PUFA patterns may be needed. In a study of older adults, Fohtung et al. (2017) did not find a significant association between total hip or femoral neck BMD and incident hip fracture (HF) in women. Among men, however, there was evidence of a significant association and also effect of modification by race. Lower total hip BMD was independently associated with higher HF risk in nonblack men, but lower risk in black men. Findings were similar for femoral neck BMD, although these fell short of statistical significance, possibly reflecting dual-energy x-ray absorptiometry's (DXA's) higher precision errors for BMD at the femoral neck than the total hip. The statistically significantly higher risk of HF fracture with lower BMD of the hip in nonblack men persisted after exclusion of subclinical HF for N-terminal pro B-type natriuretic peptide 
(NT-proBNP) at the level $\geq 190 \mathrm{pg} / \mathrm{mL}$ ), although the lower risk of HF with lower BMD in black men was attenuated and no longer significant. These findings suggest that screening for osteoporosis in whites may potentially offer opportunities for earlier detection and prevention of HF, at least in men (Fohtung et al., 2017). Among older adults, lower total hip BMD was associated with higher HF risk in nonblack men but lower risk in black men, with no evidence of an association in women. Further research is needed to replicate these findings and to study potential underlying pathways. Also, Virtanen et al. (2010) investigated the association of fish and EPA+DHA consumption with BMD and hip fracture and whether high LA intake, the major dietary n-6 PUFA, modifies the associations. The result showed that high LA intake did not modify these associations. In this large prospective cohort of older adults, fish consumption was associated with very small differences in BMD and had no association with hip fracture risk. In another study, Weiss et al. (2005) investigated the association between the ratio of dietary n- 6 to n-3 fatty acids and BMD in 1532 community-dwelling men and women aged 45-90 years of age. They concluded that a higher ratio of n-6 to n-3 fatty acids is associated with lower BMD at the hip in both sexes, therefore suggesting that the relative amounts of dietary PUFAs may play a vital role in preserving skeletal integrity in old age. The results of these studies point to unclear association of BMD with n-3 fatty acid intake and therefore the need for further research.

\section{Role of Lipids in Eye Health}

Eye diseases are the second most prevalent pathologies after the age of 65 years (Wang and Daggy, 2017). Age-related macular degeneration (AMD) is the leading cause of irreversible vision loss all over the world (Jun et al., 2019).-Recent data indicate that lipid nutrients may prevent eye diseases, especially AMD (Bretillon et al., 2010; Jun et al., 2019). Lipids account for about $25 \%$ of the dry matter in the neuroretina, with phospholipids being the prominent lipids therein (more than 85\%), while cholesterol is present as free cholesterol (at 10\%), and to a lesser extent as cholesteryl esters (less than $2 \%$ ) (O'Brien and Sampson, 1965; AIIMS, 2018). Phospholipids are present in considerable quantity in the outer segment of rods and cones. Decosahexaenoic acid is the main long-chain PUFA in 
the phospholipids of the neuroretina, with $12-20 \%$ of the FAs in human and more than $30 \%$ in rodent (Wang and Daggy, 2017). It is involved in the phototransduction pathway by enhancing the ability of the photopigment rhodopsin to undergo the transition to the active form. It seems that modifying the dietary intake of omega-3 fatty acids may have consequences on the level of DHA in the retina and possibly its functioning (AIIMS, 2018). Inflammation plays a significant role in the disease process of dry eye disease (DED) and AMD. Lipid mediators, including eicosanoids and SPMs derived from membrane fatty acids, are the key players in the inflammatory process and its resolution (Wang and Daggy, 2017). In addition to the well-established beneficial effects on reducing the risk of cardiovascular diseases, EPA and DHA have now emerged as being important substrates for the production of SPMs that can bring on the ultimate resolution of the inflammatory process and may modulate angiogenesis, which is crucial in both corneal and choroidal neovascularization, the pathological process involved in certain forms of DED and AMD, respectively (Wang and Daggy, 2017). Even though EPA and DHA are not considered to be "essential" fatty acids, due to the potential but inefficient conversion of $\alpha$-linolenic acid to EPA and DHA in the human body, the only way to get sufficient EPA and DHA is through dietary intake. Based on the 03I (content of EPA + DHA in RBC membranes expressed as percentage of total fatty acid), the dosage recommendation for cardioprotective effects of $\mathrm{EPA}+\mathrm{DHA}(1 \mathrm{~g} /$ day) can also be applied to reduce the risk of neovascular AMD progression and can benefit DED patients, especially people with low $n-3$ fatty acid status (Wang and Daggy, 2017). DED is defined as a "multifactorial disease of the tears and ocular surface that results in symptoms of discomfort, visual disturbance, and tear film instability with potential damage to the ocular surface (Boudouin et al., 2016)". It is accompanied by increased osmolarity of the tear film and sub-acute inflammation of the ocular surface. The ocular surface (cornea, conjunctiva, and accessory lacrimal glands), meibomian glands (specific sebaceous glands of the eyelid margin, which produce the outer lipid film of the tear film), the main lacrimal gland, and the innervations between them form a functional unit. Any or all of these structures may be affected in DED. Recent studies have shown that dry eye is an inflammatory disease that has many features in common with autoimmune disease (Wang and Daggy, 2017; Boudouin et al., 2016). Among recently found ocular 
dystrophies, Fuchs' endothelial corneal dystrophy (FECD) is a relatively frequent degenerative corneal condition confined to the corneal endothelium, characterized by subsequent changes in cell morphology, as well as deposits of collagen in the Descemet membrane and apoptosis (Borderie et al., 2000) and endothelial cell loss that is inherited in an autosomal dominant pattern with 100\% penetrance with variable expressivity (Bahn et al., 1984). Fuchs' endothelial corneal dystrophy is associated with blurred vision, which is caused by the corneal swelling from defects in the inner corneal layer, the corneal endothelium (Zhang and Patel, 2015). The disease typically presents with its clinical characteristics at some point between the 5th and 6th decades of life and can vary from macroscopic guttae producing light scattering to a decrease in visual quality, tochronic, and full thickness corneal oedema (Zhang and Patel, 2015). Currently, the disease is considered to be a multifactorial condition, in which genetic (Gottsch et al., 2005) and environmental factors, such as UV light-induced oxidative stress (Azizi et al., 2011), contribute to its onset. Lipids are showing increasing promise as biomarkers for diseases such as FECD indicating increasing clinical relevance. Immunomodulation through the EFAs has shown promising results as therapeutic molecules (Rowsey and Karamichos, 2017). However, further studies concerning the role of lipids and the exact mechanisms of action are necessary. 


\section{CONCLUSION AND RECOMMENDATIONS}

Lipids perform numerous functions in human metabolism including-energy provision, nutrient transport and absorption enhancement, gene transcription, expression and as biomarkers. Anti-atherosclerotic effects of EPA include antiplatelet aggregation, vasodilation, anti-inflammation and maintaining plasma TG levels low. Decosahexaenoic acid functions through alterations in membrane structure and function, in membrane protein function, cellular signalling and lipid mediator production. Low DHA levels have been linked to poor cognitive development and visual function, while DPA reduces platelet aggregation, improves lipid metabolism, endothelial cell migration, and resolution of chronic inflammation. The beneficial ratio of mega- 6 to omega-3 PUFAs seems to differ with the disease but a low ratio of omega- 6 to omega- 3 seems to alleviate most disease conditions. Lipids are showing increasing promise as biomarkers for a number of diseases. The roles of marine omega-3 fatty acids in eye, brain and bone health are being established, but mechanisms of action are not fully understood, especially in brain health and mental depression. How SAFAs modulate the expression of genes encoding proteins related to lipid metabolism, the role and nature of lipids that take part in gene expression and the mechanisms of action are also less understood and require further research.

\section{NOTES}

K-Ras: A gene that makes a protein that is involved in cell signaling pathways that control cell growth, cell maturation, and cell death. The natural, unchanged form of the gene is called wild-type $K$-Ras.

K-Ras4A: The $K$-Ras oncogene is mutated more frequently in human cancer than any other. The $K$-Ras transcript is alternatively spliced to give rise to two products, K-Ras $4 \mathrm{~A}$ and KRas4B, both of which are oncogenic when $K$-Ras is mutated.

Oligodendrocytes: Oligodendrocytes are a type of large glial cell found in the central nervous system. Oligodendrocytes produce the myelin sheath insulating neuronal axons (analogous to Schwann cells in the peripheral nervous system), although some oligodendrocytes (called satellite oligodendrocytes) are not involved in myelination.

Myelin: Myelin is an insulating layer, or sheath that forms around nerves, including those in the brain and spinal cord. It is made up of protein and fatty substances. The myelin sheath allows electrical impulses to transmit quickly and efficiently along the nerve cells. If myelin is damaged, the impulses slow down.

Lipid rafts: Lipid rafts are submicroscopic membrane microdomains rich in sphingomyelin and cholesterol. Two common types of lipid raft have been proposed and 
studied in neurotransmitter signalling: planar lipid rafts (also known as non-caveolar, or glycolipid, rafts) and caveolae. Lipid rafts may be organized as domains of protein-lipid and lipid-lipid interactions.

De novo synthesis: de novo synthesis refers to the synthesis of complex molecules from simple molecules such as sugars or amino acids, as opposed to recycling after partial degradation. For example, nucleotides are not needed in the diet as they can be constructed from small precursor molecules such as formate and aspartate. Methionine, on the other hand, is needed in the diet because while it can be degraded to and then regenerated from homocysteine, it cannot be synthesized de novo.

G-protein: G proteins, also known as guanine nucleotide-binding proteins, are a family of proteins that act as molecular switches inside cells, and are involved in transmitting signals from a variety of stimuli outside a cell to its interior. Their activity is regulated by factors that control their ability to bind to and hydrolyze guanosine triphosphate (GTP) to guanosine diphosphate (GDP). When they are bound to GTP, they are 'on', and, when they are bound to GDP, they are 'off'. G proteins belong to the larger group of enzymes called GTPases.

Protein kinase C: Protein kinase $\mathrm{C}$ represents a family of serine/threonine kinases that belong to the AGC (cAMP-dependent, cGMP-dependent, and protein kinase C) superfamily of protein kinases.

IP3: In higher eukaryotes, PLC enzymes interact with cellular membranes to preferentially hydrolyze the minor membrane component phosphatidylinositol-4,5-bisphosphate ( $\mathrm{PIP}_{2}$ ), generating the second messengers inositol-1,4,5-triphosphate $\left(\mathrm{IP}_{3}\right)$ and $\mathrm{DAG}$. $\mathrm{IP}_{3}$ is essential for increasing intracellular $\mathrm{Ca}^{2+}$, while DAG activates protein kinase $\mathrm{C}(\mathrm{PKC})$ GTPase-activating proteins (GAPs): GTPases are a large family of hydrolase enzymes that bind to the nucleotide guanosine triphosphate (GTP) and hydrolyze it to guanosine diphosphate (GDP). The GTP binding and hydrolysis takes place in the highly conserved G domain common to many GTPases.

Proliferator-activated receptor $\boldsymbol{\beta}$ ( $\boldsymbol{\beta P P A R}$ ): Peroxisome proliferator-activated receptors (PPARs) are ligand-activated transcription factors of nuclear hormone receptor superfamily comprising of the following three subtypes: PPAR $\alpha, \operatorname{PPAR} \gamma$, and PPAR $\beta / \delta$. Activation of PPAR- $\alpha$ reduces triglyceride level and is involved in regulation of energy homeostasis. Activation of PPAR- $\gamma$ causes insulin sensitization and enhances glucose metabolism, whereas activation of PPAR- $\boldsymbol{\beta} / \boldsymbol{\delta}$ enhances fatty acids metabolism. Thus, PPAR family of nuclear receptors plays a major regulatory role in energy homeostasis and metabolic function.

Peroxisome proliferator-activated- $\alpha$ receptor (PPAR- $\alpha$ ): Peroxisome proliferatoractivated receptors (PPARs) are ligand-activated transcription factors of nuclear hormone receptor superfamily comprising of the following three subtypes: PPAR $\alpha, \operatorname{PPAR} \gamma$, and PPAR $\beta / \delta$. Activation of PPAR- $\alpha$ reduces triglyceride level and is involved in regulation of energy homeostasis.

Hormone sensitive lipase (HSL): Hormone-sensitive lipase (HSL) is an intracellular neutral lipase that is capable of hydrolyzing triacylglycerols, diacylglycerols, monoacylglycerols, and cholesteryl esters, as well as other lipid and water soluble substrates. HSL activity is regulated post-translationally by phosphorylation and also by pretranslational mechanisms. The enzyme is highly expressed in adipose tissue and 
steroidogenic tissues, with lower amounts expressed in cardiac and skeletal muscle, macrophages, and islets.

Lipoprotein lipase (LP): Lipoprotein lipase plays a critical role in breaking down fat in the form of triglycerides, which are carried from various organs to the blood by molecules called lipoproteins. When lipoprotein lipase breaks down triglycerides, the fat molecules are used by the body as energy or stored in fatty tissue for later use.

Fatty acid binding protein 3 (fabp3): Intracellular lipid chaperones known as fatty acidbinding proteins (FABPs) are a group of molecules that coordinate lipid responses in cells and are also strongly linked to metabolic and inflammatory pathways. FABPs are abundantly expressed 14-15 kDa proteins that reversibly bind hydrophobic ligands, such as saturated and unsaturated long-chain fatty acids, eicosanoids and other lipids, with high affinity. One of these FABPs is fabp3.

Liver X receptor (LxR): The LXRs, LXR $\alpha$ and LXR $\beta$ are recognized to be the central regulators of cholesterol and lipid metabolism in mammals. $\mathrm{LXR} \alpha$ is highly expressed in liver tissue and with lower levels present in adipose tissue, intestine, kidney, and splenic tissues, whereas LXR $\beta$ is expressed in many more tissues. Both LXR $\alpha$ and LXR $\beta$ isoforms preferentially bind with their heterodimeric partner RXR, LXR/RXR heterodimers then bind to LXR response elements in the promoters of their target genes and activate target gene expression.

Nuclear factor-kappa nuclear factor-kappaB (NFkappaB) or NFкB): is protein transcription factor that is considered to be a regulator of innate immunity. The NF- $\mathrm{B}$ signaling pathway links pathogenic signals and cellular danger signals thus organizing cellular resistance to invading pathogens. A plethora of studies have shown that NF- $\kappa \mathrm{B}$ is a network hub responsible for complex biological signaling. It seems to contribute substantially to cell survival, cell death, and the regulation of function and/or disease. Sterol regulatory element binding proteins (SREBPs): Sterol regulatory element binding proteins (SREBPs) are a family of transcription factors that regulate lipid homeostasis by controlling the expression of a range of enzymes required for endogenous cholesterol, fatty acid (FA), triacylglycerol and phospholipid synthesis.

Guanine exchange factor proteins (GEFs) and GTPase: Guanine nucleotide exchange factors (GEFs) and GTPase-activating proteins (GAPs) regulate the activity of small guanine nucleotide-binding $(G)$ proteins to control cellular functions. In general, GEFs turn on signaling by catalyzing the exchange from G-protein-bound GDP to GTP, whereas GAPs terminate signaling by inducing GTP hydrolysis. GEFs and GAPs are multidomain proteins that are regulated by extracellular signals and localized cues that control cellular events in time and space. Recent evidence suggests that these proteins may be potential therapeutic targets for developing drugs to treat various diseases, including cancer.

Peripheral blood mononuclear cells (PBMCs): A peripheral blood mononuclear cell (PBMC) is any peripheral blood cell having a round nucleus. These cells consist of lymphocytes (T cells, B cells, NK cells) and monocytes, whereas erythrocytes and platelets have no nuclei, and granulocytes (neutrophils, basophils, and eosinophils) have multi-lobed nuclei.

Lipidome: The lipidome is comprised of all of the biomolecules defined as lipids.

'Lipidomics' is theoretically the comprehensive analysis of all of its components, although in practice it usually encompasses a selected (but relatively large) number of lipids in a 
manner that has the likelihood of providing useful information about a biological process, organelle or entire organism(s), pathological state or other related subject of interest. Specialized pro-resolving mediators (SPMs): are a large and growing class of cell signaling molecules formed in cells by the metabolism of polyunsaturated fatty acids by one or a combination of lipooxygenase, cyclooxygenase, and cytochrome P450 monooxygenase enzymes.

ApoB-48: Apolipoprotein B is the primary apolipoprotein of chylomicrons, VLDL, Lp(a), IDL (intermediate density lipoprotein), and LDL (commonly known as "bad cholesterol"), which is responsible for carrying fat molecules (lipids) including cholesterol around the body to all cells within all tissues. While all the functional roles of ApoB within the LDL (and all larger) particles remain somewhat unclear, it is the primary organizing protein (of the entire complex shell enclosing/carrying fat molecules within) component of the particles and is absolutely required for the formation of these particles. The other critically important apolipoprotein is ApoB-100.

CM: Chylomicrons are the largest lipoproteins, with diameters of 75-600 nanometres (nm; $1 \mathrm{~nm}=10^{-9}$ metres). They have the lowest protein-to-lipid ratio (being about 90 percent lipid) and therefore the lowest density. Chylomicrons are synthesized by the absorptive cells of the intestinal lining and are secreted by these cells into the lymphatic system which joins the blood circulation at the subclavian vein. They transport lipids from the intestinal tract to body cells for further metabolism.

Citing this article: Lokuruka, M.N.I. (2021). Roles of lipids in health and disease. Journal of Food and Nutritional Sciences Research; 3(1), p ----. Doi. --------------. 


\section{REFERENCES}

Afman, L., Milenkovic, D. \& Roche, H.M.. (2014). Nutritional aspects of metabolic inflammation in relation to health-insights from transcriptomic biomarkers in PBMC of fatty acids and polyphenols. Mol Nutrition Food Res. 58, 1708 -

20. https://doi.org/10.1002/mnfr.201300559.

Afman, L.A. \& Muller, M. (2012). Human nutrigenomics of gene regulation by dietary fatty acids. Progress Lipid Res. 51, 63-70. https://doi.org/10.1016/j.plipres.2011.11.005.

Aiims, RK. Lipids and the eye. 2018; Found at:

https://aiimsrishikesh.edu.in/newwebsite/wpcontent/uploads/2018/09/770 Lipids Eye.pdf. Accessed 10 th July, 2021.

Alhouayek, M., Ameraoui, H. \& Muccioli, G.G. (2021). Bioactive lipids in inflammatory bowel diseases - From pathophysiological alterations to therapeutic opportunities.

Biochimica et Biophysica Acta (BBA) - Molecular and Cell Biology of Lipids 1866 (2), 158854.

Astorg, P., Bertrais, S., Laporte, F., Arnault, N., Estaquio, C., Galan, P., Favier, A. \& Hercberg, S. (2008). Plasma n-6 and n-3 polyunsaturated fatty acids as biomarkers of their dietary intakes: a cross-sectional study within a cohort of middle-aged French men and women. Eur. J. Clin. Nutrition 62, 115561.

https://doi.org/10.1038/sj.ejcn.1602836.

Ayisi, L.C., Cheng, C., Cheng, Y. \& Jinliang, Z. (2017). Genes, transcription factors and enzymes involved in lipid metabolism in fin fish. Agri gene 7.10. doi.org/1016/j.aggene. 2017.09.006. 
Azizi, B., Ziaei, A., Fuchsluger, T., Schmedt, T., Chen, Y. \& Jurkunas, U.V. (2011). p53regulated increase in oxidative-stress-induced apoptosis in Fuchs endothelial corneal dystrophy: a native tissue model. Invest Ophthalmol Vis Sci. 52(13), 92919297.

Bae, Y.S., Cantley, L.G., Chen, C.S. \& Kim, S.R. (1998). Activation of phospholipase C-gamma by phosphatidylinositol 3,4,5-trisphosphate. J. Biol. Chem. 273(8), 4465-9.

Bahn, C.F., Falls, H.F., Varley, G.A, Meyer, R.F., Edelhauser, H.F. \& Bourne, W.M. (1984). Classification of corneal endothelial disorders based on neural crest origin. Ophthalmology. 91(6), 558-563.

Basil, M.C. \& Levy, B.D. (2016). Specialized pro-resolving mediators: endogenous regulators of infection and inflammation. Nat. Rev. Immunol. 16, 51-67. doi:10.1038/nri.2015.4.

Baudouin, C., Messmer, EM., Aragona, P., Geerling, G., Akova, Y.A., Benitez-Del-Castillo J., Boboridis, K.G., Jesús Merayo-Lloves., Rolando, M. \& Labetoulle, M. (2016). Revisiting the vicious circle of dry eye disease: a focus on the pathophysiology of meibomian gland dysfunction. Br. J. Ophthalmol. 100:300-306.

Borderie, V.M., Baudrimont, M., Vallee, A., Ereau, T.L., and Gray, F., Laroche, L. Corneal endothelial cell apoptosis in patients with Fuchs' dystrophy. (2000). Invest. Ophthalmol. Vis. Sci. 2000; 41(9), 2501-2505.

Bretillon L, Niyazi Acar, Olivier Berdeaux, Alain Bron, Creuzot-Garcher, C. (2010). Lipid Nutrition and eye health. Lipid Technol. 2010; https://doi.org/10.1002/lite.201000028. 
Bohdanowicz, M. \& Grinstein, S. (2013). Role of phospholipids in endocytosis, phagocytosis and macropinicytosis. Physiology Reviews Role of Phospholipids in Endocytosis, Phagocytosis, and Macropinocytosis. Physiological Reviews 93(1), 69-106. Found at: https://doi.org/10.1152/physrev.00002.2012. Accessed 15 ${ }^{\text {th }}$ October, 2021.

Bougarne, N., Weyers, B., Desmet, J., Deckers, J,, Ray, DW., Staels, B. \& De Bosscher, K. (2018). Molecular Actions of PPARa in Lipid Metabolism and Inflammation. Endocrine Reviews 39, 760 - 802.

Bourre, J.M. (2004). Roles of unsaturated fatty acids (especially omega-3 fatty acids) in the brain at various ages and during ageing. J. Nutr. Health Aging 8(3), 163-74.

Bouwens, M., Afman, L.A. \& Müller, M. (2007). Fasting induces changes in peripheral blood mononuclear cell gene expression profiles related to increases in fatty acid $\beta$ oxidation: functional role of peroxisome proliferator-activated receptor $\alpha$ in human peripheral blood mononuclear cells. Am. J. Clin. Nutrition 86, 1515-23.

Bouwens, M., van de Rest, O., Dellschaft, N., Bromhaar, M.G., de Groot, L.C.P.G.M., Geleijnse, J.M., Müller, M. \& Afman, L.A. (2009). Fish-oil supplementation Induces antiinflammatory gene expression profiles in human blood mononuclear cells. Amer. J. Clin. Nutr. 90(2), 415-424, Found at: https://doi.org/10.3945/ajcn.2009.27680.

Bravo-Ruiz, I., Medina, M.Á. \& Martínez-Poveda, B. (2021). From Food to Genes: Transcriptional Regulation of Metabolism by Lipids and Carbohydrates. Nutrients 13, https://doi.org/10.3390/ nu13051513.

Brown, M.S. \& Goldstein, J.L. (1997). The SREBP pathway: regulation of cholesterol Metabolism by proteolysis of a membrane-bound transcription factor. Cell 89, 33140. 
Bruce, K.D., Zsombok, A. \& Heckel, R.E. (2017). Lipid Processing in the brain: a Key Regulator of systemic metabolism. Front. Endocrinol. https://doi.org/10.3389/fendo.2017.00060.

Burdge, G.C. \& Calder, P.C. (2005). Conversion of alpha-linolenic acid to longer-chain Polyunsaturated fatty acids in human adults. Reprod. Nutr. Dev. 45, 581-597. Caimari, A., Oliver, P., Keijer, J. \& Palou, A. (2010). Peripheral blood mononuclear cells as a model to study the response of energy homeostasis-related genes to acute changes in feeding conditions. Omics: a Journal of Integrative Biology 14, 129-41.

Cermenati, G., Mitro, N., Audano, M., Melcangi, R.C., Crestani, M., De Fabiani E. \& Caruso D. (2015). Lipids in the nervous system: from biochemistry and molecular biology to patho-physiology. Biochim Biophys Acta 851(1), 51-60. doi:

10.1016/j.bbalip.2014.08.011.

Chang, C.Y., Ke, D.S. \& Chen, J.Y. (2009). Essential fatty acids and human brain. Acta Neurol Taiwan 18(4), 231-41.

Chiurchiu, V, Leuti, A. \& Maccarone M. (2018). Bioactive lipids and chronic inflammation: Managing the fire within. Front. Immunol. Found at: https://doi.org/10.3389/fimmu.2018.00038.

Chiurchiù, V., Rapino, C., Talamonti, E., Leuti, A., Lanuti M., Gueniche, A., Jourdain, R., Breton, L. \& Maccarrone, M. (2016). Anandamide suppresses proinflammatory T cell responses in vitro through type-1 cannabinoid receptor-mediated mTOR inhibition in human keratinocytes. J Immunol 197, 3545-53. doi:10.4049/jimmunol.1500546. Accessed on 9th Nov 2021.

Clària, J. (2016). Role of bioactive lipid mediators in obese adipose tissue inflammation and 
endocrine dysfunction; Molecular and Cellular Endocrinology. 20, 16.

Clària, J., Nguyen, B.T., Madenci, A.L., Ozaki, C.K, \& Serhan, CN. (2013). Diversity of

Lipid mediators in human adipose tissue depots. Am. J. Cell Physiol. 304(12), C1141C1149. doi:10.1152/ajpcell.00351.2012.

Corwin, R.L. (2003). Effects of dietary fats on bone health in advanced age. Prostaglandins Leukot Essent Fatty Acids 68, 379-386. Accessed 10th March, 2021.

Cruz, A.L.S., de Barreto, E.A., Narayana, P., Fazolini, B., João, P., Viola, B. \& Bozza, PT. (2020). Lipid droplets: platforms with multiple functions in cancer hallmarks. Cell Death Dis. 11, 105. https://doi.org/10.1038/s41419-020-2297-3. Accessed 9th Nov 2021.

de Carvalho, C.C.C.R. \& Caramujo, M.J. (2018). The Various Roles of Fatty Acids. Molecules 23(10), 2583. doi:10.3390/molecules23102583.

Del Gobbo, L.C., Fumiaki, Imamura., Stella, Aslibekyan., Matti, Marklund., Jyrki

K. Virtanen., Maria, Wennberg., Mohammad Y. Yakoob., Stephanie

E. Chiuve., Luicito dela Cruz,. Alexis C. Frazier-Wood., Amanda

M. Fretts., Eliseo, Guallar., Chisa, Matsumoto., Kiesha, Prem., Tosh, Tanaka., Jason, H., Y. Wu, Xia Zhou., Helmer, C., Ingelsson, E., Yuan., J-M., Barberger-Gateau, P., Campos, H., Chaves, P.H.M., Djoussé, L., Giles, G.G., Gómez-Aracena, J., Hodge, A., Hu, F.B., Jan-Jansson, J-H., Johansson, I., Khaw, K-T., Koh, W-P., Lemaitre, R.N., Lind, L., Luben, R.N., Rimm, E.B., Risérus, U., Samieri, C., Franks, PW., Siscovick, D.S., Stampfer, M., Steffen, L.M., Steffen, B.T., Tsai, M.Y., van Dam, R.M., Voutilainen, S., Willett, W.C., Woodward, M. \& Mozaffarian, D. for the Cohorts for Heart and Aging Research in Genomic Epidemiology (CHARGE). Fatty Acids and Outcomes Research 
Consortium (FORCe). (2016). JAMA Intern Med. 176(8), 1155-1166.

doi:10.1001/jamainternmed.2016.2925.

de Kroon, A.I., Rijken, P.J.\& De Smet, C.H. (2013). Checks and balances in membrane phospholipid class and acyl chain homeostasis, the yeast perspective. Prog Lipid Res. 52(4), 374-94.

De Lorenzo, A., Soldati, L., Sarlo, F., Calvani, M., Di Lorenzo, N. \& Di Renzo. L. (2016).

New obesity classification criteria as a tool for bariatric surgery indication. World J. Gastroenterol. 22(2), 681-703. doi: 10.3748/wjg.v22.i2.681.

de Mello, V.D.F., Kolehmanien, M., Schwab, U., Pulkkinen, L, \& Uusitupa. M. (2012). Gene expression of peripheral blood mononuclear cells as a tool in dietary intervention studies: what do we know so far? Mol. Nutrition Food Res. 56, 1160-72.

Desvergne, B., Michalik, L. \& Wahli, W. (2006). Transcriptional regulation of metabolism. Physiol. Rev. 86, 465-514. https://doi.org/10.1152/physrev.00025.2005.

Dhama, K., Latheef, S.K., Dadar, M., Samad, H.A., Munjal, A., Khandia, R., Karthik, K., Tiwari R., Yatyoo, M.I., Bhatt, P., Chakraborty, S., Singh, K.P., Iqbal, H.M.N., Chaicumpa, Ma. \& Joshi, S.K. (2019). Biomarkers in Stress Related Diseases/Disorders: Diagnostic, Prognostic, and Therapeutic Values-A review. Frontiers in Molecuar Biosciences. https://doi.org/10.3389/fmolb.2019.00091. Ebrahimi, M., Rajion, M.A., Jafari, S., Jahromi, M.F., Oskoueian, E., Sazili, A.Q., Goh, Y.M. \& Ghaffari, M.H. (2019). Correction: Effects of dietary n-6: $n$-3 polyunsaturated fatty acid ratios on meat quality, carcass characteristics, tissue fatty acid profiles, and expression of lipogenic genes in growing goats. PLoS ONE 14(9), e0222678. https://doi.org/10.1371/journal.pone.0222678. 
Georgiadi, A. \& Kersten, S. (2012). Mechanisms of gene regulation by fatty acids. Adv Nutrition (Bethesda, Md). 3, 127-34. https://doi.org/10.3945/an.111.001602.

Ghosh, A. \& Nishtala, K. (2017). Biofluid lipidome: a source for potential diagnostic biomarkers. Clin Trans Med 6, 22. https://doi.org/10.1186/s40169-017-0152-7.

Goni, F.M. (2014). The basic structure and dynamics of cell membranes: an update of the Singer-Nicolson model. Biochim Biophys Acta. 1838(6), 1467-76.

Gottsch, J.D., Zhang, C., Sundin, O.H., Bell, W.R., Stark, W.J., Green. \& Fuchs, WR. (2005). corneal dystrophy: aberrant collagen distribution in an L450W mutant of the COL8A2 gene. Invest. Ophthalmol Vis. Sci. 2005;46(12):4504-4511.

Gutiérrez, S., Svahn, S.L. \& Johansson, M.E. (2019). Effects of Omega-3 Fatty Acids on Immune Cells. Int J Mol Sci. 20(20), 5028. doi: 10.3390/ijms20205028.

Fohtung, R.B., Brown, D.L., Koh, T.W.J., Bartz, H.M., Carbone, L.D., Civitelli, R., Stein, P.K., Chaves, P.H.M., Kestenbaum, B.R. \& Kizer, JR. (2003). Bone Mineral Density and Risk of Heart Failure in Older Adults: The Cardiovascular Health Study.

J. Amer. Heart Assoc. 6(3). https://doi.org/10.1161/JAHA.116.004344.

Hannun, Y.A. \& Bell, R.M. (1989). Regulation of protein kinase C by sphingosine lysosphingolipids. Clin Chim Acta. 185(3), 333-45.

Hardie, R.C. \& Muallem, S. (2009). Lipids in Ca2+ signalling-an introduction. Cell Calcium. $45,517-20$.

Hayes, C.E. \& Ntambi, J.M. (2020). Multiple Sclerosis: Lipids, Lymphocytes, and Vitamin D. Immunometabolism 2(3), e200019. doi: 10.20900/immunometab20200019. Hussain, G., Anwar, H., Rasul, A., Imran, A., Qasim, M., Zafar, S., Imran, M., Syed Kashif, S., Kamran, S., Aziz, N., Razzaq, A., Waseem-Ahmad, Shabbir, A., Iqbal, J., Baig, S.M., 
Ali, M., Gonzalez de Aguilar, J-L., Sun, T., Muhammad, A \& Umar. A.M. (2020). Lipids as biomarkers of brain disorders, Critical Reviews in Food Science and Nutrition 60, 3, 351-374. DOI: 10.1080/10408398.2018.1529653.

Hussain, G., Schmitt, F., Loeffler, J.P., de Aguilar, J.L.G. (2013). Fatting the brain: a brief of recent research. Front. Cell Neurosci. 7, 1-14. Available from: http://journal.frontiersin.org/article/10.3389/fncel.2013.00144/abstract. Accessed 23rd July, 2021.

Jenkins-Kruchten, A.E, Bennaars-Eiden, A., Ross, JR, Shen, W.J., Kraemer, F.B, \& Bernlohr, D.A. (2003). Fatty acid-binding protein-hormone-sensitive lipase interaction. Fatty acid dependence on binding. J. Biol. Chem. 278(48), 47636-43. doi: 10.1074/jbc.M307680200.

Jump, D.B. (2018). Fatty Acid Regulation of Gene Transcription, Critical Reviews in Clinical Laboratory Sciences 41(1), 41-78. DOI: 10.1080/10408360490278341

Jump, D.B. (2008). N-3 polyunsaturated fatty acid regulation of hepatic gene transcription. Curr. Opin. Lipidol. 19(3), 242-7. doi: 10.1097/MOL.0b013e3282ffaf6a.

Jun, S., Datta, S., Wang, L., Pegany, R., Cano, M., Handa, JT. (2019). The impact of lipids, Lipid oxidation, and inflammation on AMD, and the potential role of miRNAs on lipid metabolism in the RPE. Exp Eye Res. 181:346-355. doi:10.1016/j.exer.2018.09.023.

Karagianni, P. \& Talianidis, I. (2015). Transcription factor networks regulating hepatic fatty acid metabolism. Biochimica et Biophysica Acta (BBA)-Molecular and Cell Biology of Lipids 1851(1), 2-8. https://doi.org/10.1016/j.bbalip.2014.05.001. Accessed 15th Nov 2021. 
Karpen, S. (2002). Nuclear receptor regulation of hepatic function. J. Hepatology 36, 832850.

Kiens, B., Roepstorff, C., Glatz, J.F., Bonen, A., Schjerling, P., Knudsen, J, \& Nielsen, J.N. (1985). Lipid-binding proteins and lipoprotein lipase activity in human skeletal muscle: influence of physical activity and gender. J. Appl. Physiol. (1985). 97(4), 1209-18. doi: 10.1152/japplphysiol.01278.2003.

Korade Z, Kenworthy AK. (2008). Lipid rafts, cholesterol, and the brain. Neuropharmacology. 55(8), 1265-73.

Kumar, V., Abbas, A. \& Aster, J. (2014). Robbins \& Cotran Pathologic Basis of Disease. IX ed. Amsterdam: Elsevier. Accessed on 9th Nov 2021.

Larrouy-Maumus, G. (2019). Lipids as Biomarkers of Cancer and Bacterial Infections. Curr. Med. Chem. 26(11), 1924-1932. doi: 10.2174/0929867325666180904120029.

Larsen, S.V., Holven, K.B., Ottestad, I., Kine, N., Dagsland, M., Myhrstad, C.W.\& Stine, M.U. (2018). Correction to: Plasma fatty acid levels and gene expression related to lipid metabolism in peripheral blood mononuclear cells: a cross-sectional study in healthy subjects. Genes Nutr 13, 9. https://doi.org/10.1186/s12263-018-0600-z. Leyrolle, Q., Decoeur, F., Briere, G., Amadieu, C., Quadros, ARAA., Voytyuk I., Lacabanne, C., Benmamar-Badel, A., Bourel, J., Aubert, A., Sere, A., Chain, F., Schwendimann, L., Matrot, B., Bourgeois, T., Grégoire, S., Leblanc, J.G., De Moreno, De., Leblanc, A., Langella, P., Fernandes, G.R., Bretillon, L., Joffre, C., Uricaru, R., Thebault. P., Gressens, P., Chatel, J.M., Layé, S. \& Nadjar, L.S. (2021). Maternal dietary omega-3 
Deficiency worsens the deleterious effects of prenatal inflammation on the gut-brain axis in the offspring across lifetime. Neuropsychopharmacology 46(3), 579-602. Doi: 10.1038/s41386-020-00793-7.

Li, Z.L. \& Buck. M. (2017). Computational Modeling Reveals that Signaling Lipids Modulate The Orientation of K-Ras4A at the Membrane Reflecting Protein Topology. Structure 25(4), 679-89. e2.

Linton, M.F., Yancey, P.G., Davies, S.S., Jerome, W.G., Linton E.F, Song, W.L, Doran, A.C., Vickers, K.C., Feingold, K.R., Anawalt, B., Boyce, A., Chrousos, G., de Herder, W.W., Dhatariya, K., Dungan, K., Hershman, J.M., Hofland, J., Kalra, S., Kaltsas, G., Koch, C., Kopp, P., Korbonits, M., Kovacs, C.S., Kuohung, W., Laferrère, B., Levy, M., McGee, E.A., McLachlan, R., Morley, J.E., New, M., Purnell, J., Sahay, R., Singer, F., Sperling, M.A., Constantine, A., Stratakis, A.S., Trence, D.L. \& Wilson, D.P. (2000). The Role of Lipids and Lipoproteins in Atherosclerosis. In: Feingold, K.R., Anawalt, B., Boyce, A. (editors). Endotext [Internet]. South Dartmouth (MA): MDText.com, Inc. 2000. Available from: https://www.ncbi.nlm.nih.gov/books/NBK343489/. Accessed 12th June, 2021.

Lopategi, A., López-Vicario, C., Alcaraz-Quiles J., García-Alonso, V., Rius, B., Titos, E., Liu, HQ., Qiu, Y., Mu, Y., Zhang, X.J., Liu, L., Hou, X.H., Zhang, L., Xu, X.N., Ji, A.L., Cao, R., Yang, R.H. \& Wang, F. (2013). A high ratio of dietary n-3/n-6 polyunsaturated fatty acids improves obesity-linked inflammation and insulin resistance through suppressing activation of TLR4 in SD rats. Nutr Res. 33(10), 84958. doi: 10.1016/j.nutres.2013.07.004.

Makinen, M., Halvorsen, B., Retterstøl, K., Kirkhus, B, Granlund, L, Holven, K.B. \& Ulven, 
S.M. (2011). Effect of the fat composition of a single high-fat meal on inflammatory Markers in healthy young women. Br. J. Nutr. 106, 1826-35. https://doi.org/10.1017/s0007114511002510.

Myhrstad, M.C.W., Narverud, I., Telle-Hansen, V.H., Karhu, T., Lund, D.B, Herzig, KH., Natarajan, R. \& Nadler, J.L. (2004). Lipid Inflammatory Mediators in Diabetic Vascular Disease. Arteriosclerosis, Thrombosis, and Vascular Biology. 24: 15421548. https://doi.org/10.1161/01.ATV.0000133606.69732.4c

Navarini, L., Bisogno, T., Paolo, D., Margiotta, E., Piccoli, A., Angeletti, S., Laudisio, A., Ciccozzi, M., Afeltra, A. \& Maccarrone, M. (2018). Role of the Specialized Proresolving Mediator Resolvin D1 in Systemic Lupus Erythematosus: Preliminary Results. J. Immunol Res. 5264195. doi:10.1155/2018/5264195.

O'Brien, J.S. \& Sampson EL: Fatty acid and fatty aldehyde composition of the major brain lipids in normal human gray matter, white matter, and myelin. J Lipid Res 1965; 6: 545-551.

Oh, W., Abu-Elheiga, L., Kordari, P., Gu, Z., Shaikenov, T., Chirala, S.S. \& Wakil, S.J. (2005). Glucose and fat metabolism in adipose tissue of acetyl-CoA carboxylase 2 Knockout mice. Proceedings of the National Academy of Sciences, USA, 102(5), 1384-1389. Found at: https://doi.org/10.1073/pnas.0409451102.

Perdew, G.H., Vanden-Heuvel, J.P. \& Peters, J.M. (2006). Regulation of gene expression Molecular mechanisms. Totowa, NJ: Humana Press.

Prakash, B. (2021). Functional and preservative properties of phytochemicals. Elsevier, BV. Doi. https://doi.org/10.1016/C2018-0-03991-2. Accessed 12th Jun 2021. 
Poulsen, L., Sierbaek, M. \& Mandrup, S. (2012). PPARs: fatty acid sensors controlling metabolism. Semin. Cell Dev. Biol. 23(6): 631-639. Found at: https://dx.doi/org/10.1016/j.semedb.2012.01.003.

Rehfeld, J.E. (2004). Clinical endocrinology and metabolism-Cholecystokinin. Best Pract Res. Clin. Endocrinol. Metab. 18(4), 569-86.

Rehlfeld, J.E. (2017). Cholesystokinin from local gut hormone to ubiquitous messenger, Frontiers in Endocriology (Lausanne), 8, 47. doi: 10.3389/fendo.2017.00047.

Ridone, P., Grage, SL., Patkunarajah. A., Battle, A.R., Ulrich, A.S. \& Martinac, B. (2017). "Force-from-lipids" gating of mechanosensitive channels modulated by PUFAs. J. Mech. Behav. Biomed. Mater. Netherlands79, 158-67.

Rinaldin, M., Fonda. P., Giomi, L \& Kraft, D.J. (2020). Geometric pinning and antimixing in scaffolded lipid vesicles. Nat Commun 11, 4314. https://doi.org/10.1038/s41467-02017432-w. accessed 20th Nov 2021.

Roux, P.P. \& Topisirovic, I. (2018). Signaling Pathways Involved in the Regulation of mRNA Translation. Mol Cell Biol.; 38(12):e00070-18. doi:10.1128/MCB.00070-18.

Roux, P.P. \& Topisirovic, I. (2012). Regulation of mRNA translation by signaling pathways. Cold Spring Harb Perspect Biol. 2012 Nov 1;4(11):a012252. doi: 10.1101/cshperspect.a012252.

Rowsey, T.G. \& Karamichos, D. (2017). The role of lipids in corneal diseases and dystrophies: a systematic review. Clinical \& Translational Medicine 6(1), 30. doi:10.1186/s40169-017-0158-83.

Ruiz, F., Vigne, S. \& Pot, C. (2019). Resolution of inflammation during multiple sclerosis. Semin Immunopathol. 41(6),711-726. doi:10.1007/s00281-019-00765-0. 
Sambra, V., Echeverria, F., Valenzuela, A., Chouinard-Watkins, R. \& Valenzuela, R. (2021). Docosahexaenoic and Arachidonic Acids as Neuroprotective Nutrients throughout the Life Cycle. Nutrients 18, 13(3), 986. doi: 10.3390/nu13030986.

Sampath, H. \& Ntambi, J.M. (2005). Polyunsaturated fatty acid regulation of genes of lipid metabolism. Annu Rev Nutr. 25,317-40. doi: 10.1146/annurev.nutr.25.051804.101917.

Scaglia, N., Chisholm, J.W. \& Igal, R.A. (2009). Inhibition of stearoylCoA desaturase-1 inactivates acetyl-CoA carboxylase and impairs proliferation in cancer cells: role of AMPK. PLoS One. 4(8), e6812. doi: 10.1371/journal.pone.0006812.

Serhan, C.N. (2014). Pro-resolving lipid mediators are leads for resolution physiology. Nature. 510, 92-101. doi:10.1038/nature13479.

Sezgin, E., Levental. I., Mayor, S. \& Eggeling, C. (2017). The mystery of membrane organization: composition, regulation and roles of lipid rafts. Nat. Rev. Mol. Cell Biol. 18(6), 361-74.

Singh, K.P., Miaskowski, C., Dhruva, A.A., Flowers. E. \& Kober, K.M. (2018). Mechanisms and Measurement of Changes in Gene Expression. Biol. Res. Nurs. 20(4), 369-382. doi: $10.1177 / 1099800418772161$.

Shimizu, T. (2009). Lipid mediators in health and disease: enzymes and receptors as therapeutic targets for the regulation of immunity and inflammation. Ann. Rev. Pharmacol. Toxicol. 49, 123-50. doi:10.1146/annurev.pharmtox.011008.145616. Shin, A.C., Zheng, H.Y. \& Berthoud, H.R. (2009). An expanded view of energy homeostasis: Neural integration of metabolic, cognitive, and emotional drives to eat. Physiol. ehav. $97,572-80$. 
Sophie, N., Selby-Pham, B., Rosalind, B., Miller, M.K., Dunshea, F. \& Bennett, L.E. (2017). Physicochemical properties of dietary phytochemicals can predict their passive absorption in the human small intestine. Sci .Rep. 7, 193. doi: 10.1038/s41598-01701888-w. Accessed 10th September, 2021.

Stern, J.H., Rutkowski, J.M. and Scherer, P.E. (2016). Adiponectin, Leptin, and Fatty Acids in the Maintenance of Metabolic Homeostasis through Adipose Tissue Crosstalk. Cell Metabolism; 23(5): 770-784. doi:10.1016/j.cmet.2016.04.011.

Sunshine, H. \& Iruela-Arispe, M.L. (2017). Membrane lipids and cell signaling. Curr. Opin. Lipidol. 28(5), 408-413. doi:10.1097/MOL.0000000000000443.

Vachharajani, V, \& Granger, D.N. (2009). Adipose tissue: a motor for the inflammation associated with obesity. IUBMB Life. 2009;61(4):424-430. doi:10.1002/iub.169. van der Veen, J.N,, Lingrell, S., McCloskey, N., LeBlond, N.D,, Galleguillos, D., Zhao, Y.Y., Curtis, J.M., Sipione, S., Fullerton, M.D., Vance, D.E, and Jacobs, R.L. (2019). A role for phosphatidylcholine and phosphatidylethanolamine in hepatic insulin signaling. FASEB Journal; 33(4):5045-5057. doi: 10.1096/fj.201802117R. van Raalte, DH., Li, M., Pritchard, P.H. \& Wasan, K.M. (2004). Peroxisome proliferatoractivated receptor (PPAR)-alpha: a pharmacological target with a promising future. Pharm. Res. 21(9), 1531-8. doi:10.1023/b:pham.0000041444.06122.8d. Vairappan, B. (2016). Chapter 15-Cholesterol Regulation by Leptin in Alcoholic Liver Disease. In: Molecular Aspects of Alcohol and Nutrition. A Volume in the Molecular Nutrition Series, 187-200. 
Virtanen, J.K., Mozaffarian, D., Cauley, J.A., Mukamal, K.J., Robbins, J. \& Siscovick, D.S. (2010). Fish consumption, bone mineral density, and risk of hip Fracture among older adults: the cardiovascular health study. J. Bone Miner. Res., 25, 1972-1979.

Walchuk, C. \& Suh, M. (2020). Nutrition and the aging retina: A comprehensive review of the relationship between nutrients and their role in age-related macular degeneration and retina disease prevention. Adv. Food Nutr. Res. 93, 293-332.doi: 10.1016/bs.afnr.2020.04.003.

Watkins, B.A., Li, Y., Lippman, H.E. \& Feng, S. (2003). Modulatory effect of omega-3 polyunsaturated fatty acids on osteoblast function and bone metabolism. Prostaglandins Leukot Essent Fatty Acids. 68(6), 387-98. doi: 10.1016/s09523278(03)00063-2.

Weiss, L.A., Barrett-Connor, E. \& von Mühlen, D. (2005). Ratio of n-6 to n-3 fatty acids and bone mineral density in older adults: the Rancho Bernardo Study. Amer. J. Clin. Nutr. 81(4), 934-938. https://doi.org/10.1093/ajcn/81.4.934.

Yehuda, S., Rabinovitz, S. \& Mostofsky, D.I. (2005). Essential fatty acids and the brain: from infancy to aging. Neurobiol. Aging. 26, Suppl 1, 98-102. doi: 10.1016/j.neurobiolaging.2005.09.013.

Yoo, S., Kim, K., Nam, H. \& Lee, D. (2018). Discovering Health Benefits of Phytochemicals with Integrated Analysis of the Molecular Network, Chemical Properties.

Zarrouk, A., Debbabi., M, Bezine, M., Karym, E.M., Badreddine, A., Cherkaoui-Malki M., ElAyeb, M., Nasser, B., Hammami, M. \& Lizard, G. (2018). Lipid Biomarkers in Alzheimer's Disease. Curr. Alzheimer Res. 15(4), 303-312. doi: 10.2174/1567205014666170505101426. 
